\title{
Intrauterine Contraceptive Device (IUCD-Cu T380a) Migration-A Case Report
}

\author{
Subha Sivagami Sengodan*, Sumathi and Anbarasi Pandian \\ Department of Urology, India \\ *Corresponding author: Subha Sivagami Sengodan, Department of Urology, India

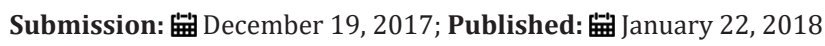

\begin{abstract}
Background: Though CU T was designed to prevent unwanted pregnancies, a number of complications are being reported. This may end up in asymptomatic course or present as an acute abdomen after migration into peritoneal cavity.

Case: Here we report a case of an intrauterine contraceptive device (IUCD-CU T380A)migration into the abdominal cavity. An open approach was used and the intrauterine contraceptive device was retrieved.
\end{abstract}

Keywords: Intrauterine contraceptive device; CU T380A; Perforation; Migration

\section{Introduction}

Intrauterine contraceptive devices were regarded as a safe effective and economic form of contraception. Possible serious complications associated with its use restricts utilisation by a large part of general population [1]. These may migrate inside peritoneal cavity and pathway for migration is via uterus or fallopian tube. In nonpregnant state spontaneous perforation may also occur with IUCD. It may perforate the wall of uterus but may remain within the myometrium. At times they may perforate the entire thickness of myometrium and either lies freely in the peritoneal cavity or more often gets embedded in the abdominal viscera [2]. But the mechanism and etiology of perforation of IUCD remains controversial. Depending on site and severity of involvement, it presents with varying abdominal symptoms and signs or may even remain asymptomatic [3]. Usually $30 \%$ cases remain asymptomatic. Even if asymptomatic they have to be retrieved to prevent further complications. Retrieval is either done by laparoscopic or open methods depending on the facilities available.

\section{Case History}

Our case reports a 22 year old female P2L2 Previous 2 LSCS, She gave history of regular menstrual cycles. Last child birth 4 months back with complaints of wound over the anterior abdominal wall in right paraumbilical region with pain for 10 days. She gave history of pus discharge for 3 days from the same site. She had no history of fever and no history of any other previous surgeries done other than LSCS. Her vitals were stable. She was afebrile with no pallor or pedal edema. CVS-S1S2 heard, RS-B/L air entry present. Per abdomen-On inspection-an irregular erythematous swelling of size $8 * 5 \mathrm{cms}$ seen over the suprapubic region extending upto right iliac fossa $3 \mathrm{~cm}$ above previous lscs scar with minimal serous discharge seen coming from it. Skin over the swelling is shiny and abdomen moves with respiration. A transverse scar(lscs) is seen inferior to the swelling and there were no dilated veins. On palpation the swelling was warm tender and indurated over the right iliac fossa region. It was immobile with diffuse borders and irregular shape of size $8 * 5 \mathrm{cms}$. Cough impulse test was negative. Per vaginal examination showed no abnormal discharge with cervix pointing downwards and uterus anteverted, normal size and fornices free. Per rectal examination showed normal fecal staining with no mass palpable. Investigations were-Hb-12gm\%, PCV-34\%, Platelet-2lakh/cu mm, RBS-104mg\%, S.urea- $24 \mathrm{mg} \%$, S.creatinine- $0.8 \mathrm{mg} \%$. USG showed abdominal wall subcutaneous edema with collection tracking into peritoneal cavity and opening into skin with cellulitic changes. Hpe report from scrapping of uterine cavity showed bundles of fibro collagenous tissues with mixed inflammatory cell infiltrates, granulation tissue with few foreign body type of giant cells seen. no granulomas made out. IMPRESSION: chronic non specific inflammation. So the case has been diagnosed as abdominal wall induration and planned to proceed with wound exploration and proceed. Intraoperatively we found that an intrauterine contraceptive device namely CU T $380 \mathrm{~A}$ was found to be dislodged into the anterior abdominal wall by perforating the uterus and migrating into the abdominal cavity.

\section{Discussion}

The incidence of IUCD migration is $1-3 / 1000$ insertions. In developing countries, IUCD forms the most common method of family planning and they are cost effective. Various devices used are CU T200, CU T multiload 250, multiload CU T375.During the past decades there has been a significant improvement in its design and content. The idea is to obtain maximum efficacy without increasing 
the adverse effects. The device is classified as open when it has got no circumscribed aperture of more than $5 \mathrm{~mm}$ so that a loop[ of intestine or omentum cannot enter and become strangulated if accidentally the device perforates through the uterus into the peritoneal cavity. Eg: Lippes loop, CuT, Multiload and progestasert. If closed devices like Grafenberg ring and Birnberg bow, accidentally enter the abdominal cavity, and they have the potential of causing strangulation of the gut and hence they are obsolete. Complete extrusion of the device through the myometrium may be aided by spontaneous uterine contraction and hydrostatic negative pressure differences between the low intraperitoneal pressure and relatively high intrauterine pressure. The migration is also aided by the contraction of the abdominal viscera like urinary bladder and intestines. Another possible mechanism is by movement of peritoneal fluid [4]. In the present case it seems that the IUCD has perforated the uterus at the time of its initial insertion and the patient has been in lactational amenorrhoea which is a risk factor for IUCD perforation due to small uterus. Perforation is often suspected or diagnosed when the IUCD string is no longer visible at the external os. Risk factors are use in nullipara, postpartum or postabortal insertion, faulty technique of insertion and irregular follow up [5]. Pelvic complications are in the form of dysmenorrhoea, pelvic inflammatory device, septic abortion and hydrosalphinx. Perforation and migration of the IUCD is one of the most rare and dreadful complication [6]. Regular self examination, investigation of persistent pain or disappearance of strings may detect migration early [7].

\section{Conclusion}

Copper containing devices have been shown to cause considerable tissue response incited with their presence [8], translocation to the peritoneal cavity may provoke peritoneal or omental adhesions, volvulus, uterocutaneous fistula and bowel perforation which involve significant morbidity. The treatment of migrated IUCD is either laparoscopy or laparotomy. A regular followup for detection of misplacement of IUCD's is stressed as it can have unusual presentation. So this case report highlights the importance of suspecting a foreign body with any abdominal wall induration or even if asymptomatic, the need for vigilance in misplaced IUCD. Plain xrays can pick up the diagnosis and exclde migration or perforation so that further complications and morbidity are prevented.USG and hysteroscopy are also useful to locate and retrieve the IUCD. Shirodhkar hook or curette is also used for removal. Laparoscopy has advantages over other procedures in locating the IUCD and full lesion assessment. Detection of asymptomatic migrated IUCD necessitates retrieval in order to discourage psychosomatic symptomatology commonly associated with forgotten devices and prevention of future grave complications.

\section{Consent}

The patient described in the paper has given their informed consent for the case report to be published.

\section{References}

1. Tinelli A, Tinelli R, Malvasi A, Cavallotti C, Tinelli FG (2010) The intrauterine device in modern contraception: still an actuality? Eur J Contracept Reprod Health Care 11(3): 197-201.

2. Koo HR, Oh YT, Kim YT, Kim SW, Kang JH (2008) Intrauterine device found in an ovarian carcinoma. J Comput Assist Tomogr 32(1): 69-71.

3. Singhal SR, Marwah DS, Paul A, Singhal SK (2010) Missed Intra Uterine Device: A Rare Indication for Appendicectomy Review of Literature. East and Central African Journal of Surgery 15(2).

4. Tunçay YA, Tunçay E, Güzin K, Oztürk D, Omurcan C (2004) Transuterine migration as a complication of intrauterine contraceptive devices:six case reports. Eur J Contracept Reprod Health Care 9(3): 194-200.

5. Gyasi-Sarpong CK, Maison PO, Morhe E, Aboah K, Appiah KA, et al. (2016) Intravesical migration of an intrauterine device. BMC Res Notes 9: 4.

6. Moulay A, Zahi M (1983) Ábdominal migration of intrauterine devices.A report of four cases removed by laparoscopy. Sem Hop 59(42): 29052909.

7. Otolorin E (1985) Management of the lost IUD. Áfrican Journal of Medicine and Medical Sciences 14(3-4): 125-129.

8. Ferchiou M, Zhioua F, Hasnaoui M, Sghaier S, Jedoui A (1995) Laparoscopic surgery of an intraperitoneal intrauterine device,'Revue Francaise de Gynecologie et d Obstetrique 90(10): 409-411. 